\title{
Efecto de la Fritura al Vacío sobre el Contenido de los Glicoalcaloides $\alpha$-Solanina y $\alpha$-Chaconina en Papa cv Botella Roja
}

\author{
Diego M. Trejo-Escobar ${ }^{{ }^{\star}}$, Misael Cortés ${ }^{2}$ y Diego F. Mejía-España ${ }^{1}$ \\ (1) Universidad de Nariño. San Juan de Pasto, Colombia. (e-mail: dmtrejoe@udenar.edu.co; \\ diegomejiaes@udenar.edu.co) \\ (2) Universidad Nacional de Colombia - Sede Medellín, Colombia. (e-mail: mcortesro@unal.edu.co)
}

\section{Resumen}

Recibido Ago. 1, 2018; Aceptado Oct. 8, 2018; Versión final Dic. 12, 2018, Publicado Ago. 2019

\begin{abstract}
Se evaluó la influencia de la fritura al vacío sobre el contenido de glicoalcaloides $\alpha$-solanina y $\alpha$-chaconina en chips de papa cv Botella Roja. Los glicoalcaloides son sustancias que afectan la salud humana, por lo que conocer su contenido en alimentos es de especial importancia. Se utilizó la metodología de superficie de respuesta con un diseño central compuesto, considerando los factores: $\Delta T$ ( $\left.T_{\text {aceite }}-T_{\text {eb. agua }}\right)\left(40-60^{\circ} \mathrm{C}\right)$, presión de vacío (30-70 kPa) y tiempo (3-7 min). Las variables dependientes son los contenidos de a-solanina y achaconina, determinadas por HPLC a partir de curvas de calibración. La papa fresca presentó contenidos de a-chaconina $\left(27,6 \pm 1,9 \mathrm{mgkg}^{-1}\right)$ y $\alpha$-solanina $\left(22,8 \pm 2,4 \mathrm{mgkg}^{-1}\right)$, los cuales están afectados por el proceso de fritura al vacío. Este le confiere una disminución del $96,2 \%$ y $100 \%$ de glicoalcaloides, respectivamente, bajo las condiciones de operación $\Delta \mathrm{T}=48,2^{\circ} \mathrm{C}\left(\mathrm{T}_{\text {aceite: }} 131,4^{\circ} \mathrm{C}\right)$, presión de vacío de 49,0 Kpay tiempo de 7 min. En este contexto, la fritura al vacío representa una tecnología efectiva en la disminución de glicoalcaloides en papa cv Botella Roja.
\end{abstract}

\section{Effect of Vacuum Frying on the Content of the Glycoalkaloids $\alpha$-Solanine and $\alpha$-Chaconine in Potato cv Botella Roja}

\begin{abstract}
The influence of vacuum frying on the content of glycoalkaloids $\alpha$-Solanine and $\alpha$-Chaconine on potato chips cv Botella Roja was evaluated. Glycoalkaloids are substances that affect human health, so its contents in food is of especial importance. The response surface methodology was used with a central composite design, considering the factors: $\Delta T$ ( $T_{\text {oil }}-T_{\mathrm{b}}$ water $)(40-60 \stackrel{\circ}{\circ} \mathrm{C})$, vacuum pressure $(30-70 \mathrm{kPa})$ and time $(3-7 \mathrm{~min})$. The dependent variables are the content of $\alpha$-Solanine and $\alpha$-Chaconine, determined by HPLC from calibration curves. The fresh potato presented contents of $\alpha$-Chaconine of $7.6 \pm 1.9 \mathrm{mgkg}^{-1}$ and $\alpha$-Solanine of $22.8 \pm 2.4$ $\mathrm{mgkg}^{-1}$, which are affected by the vacuum frying process. This gives a decrease of $96.2 \%$ and $100 \%$ of glycoalkaloids, respectively, under operating conditions $\Delta T=48.2^{\circ} \mathrm{C}$, $\mathrm{T}_{\text {oil }}=131.4{ }^{\circ} \mathrm{C}$, vacuum pressure 49.0 $\mathrm{kPa}$ and time $7 \mathrm{~min}$. In this context, vacuum frying represents an effective technology in the decrease of glycoalkalloids in potato cv Botella Roja.
\end{abstract}




\section{INTRODUCCIÓN}

En el mundo, la papa es un cultivo importante dentro de las actividades agrícolas, con impacto social y económico, principalmente en países latinoamericanos; posee alta diversidad genética, lo que se refleja en una amplia disponibilidad de variedades nativas y mejoradas, considerándose un alimento importante para la seguridad alimentaria por su alto consumo a nivel mundial. La papa es un producto básico de la canasta familiar, una porción de $100 \mathrm{~g}$ proporciona al valor diario recomendado: calorías (5\%), vitamina C (22\%), potasio (16\%), vitamina B6 (15\%), fibra (13\%), proteína (2\%), ácido fólico (10\%), magnesio (9\%) (Singh y Kaur, 2016). Por otro lado, en el género Solanum se ha identificado una amplia gama de metabolitos secundarios compuestos fenólicos, antioxidantes, lecitinas (Milner et al., 2011). El nivel promedio de consumo mundial percápita en el año 2013 fue de 34,17 kg/persona, para Europa 82,59 kg/persona; Norte América $53,78 \mathrm{~kg} /$ persona, mientras que, a nivel de Latinoamérica, para el mismo año se destaca Perú $(82,5$ $\mathrm{kg} /$ persona), Bolivia $(64,66 \mathrm{~kg} /$ persona) $(F A O, 2017)$ y Colombia para el año 2016 con $52 \mathrm{~kg} /$ persona (Fedepapa, 2016). En Colombia se cultivan diversas variedades de papa como Parda Suprema, Parda Pastusa, Diacol Capiro, ICA Nariño y Criolla, entre otras, bajo un sistema de economía campesina minifundista, en diferentes altitudes y condiciones geográficas y climáticas (Latorre-Vásquez et al., 2017), siendo su producción para el 2016 un 3,6 \% del total de productos agrícolas (FAO, 2017).

Por otro lado, se ha identificado en la papa un contenido importante de alcaloides y glicoalcaloides (Tömöskozi-Farkas et al. 2006; Milner et al., 2011). Los glicoalcaloides son compuestos biológicamente activos, que ofrecen cierta protección importantes en la defensa natural de las plantas contra insectos y hongos; sin embargo, estos llegan a ser tóxicos para la salud humana, generando perturbaciones gastrointestinales por daños en membranas y trastornos neurológicos por la inhibición de la enzima acetilcolinesterasa (Friedman, 2006; Tömösközi-Farkas et al., 2006; Cheng-Yu et al., 2018). Las papas contienen principalmente los glicoalcaloides $\alpha$-solanina y a-chaconina, aunque también pueden estar

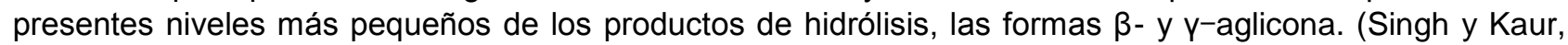
2016). Algunas especies de papa han sido reportadas con niveles de glicoalcaloides totales (GT) entre 123 a $7348 \mathrm{mgkg}^{-1}$ papa fresca (Tömösközi-Farkas et al., 2006), donde el $\alpha$-solanina y a-chaconina representan más del 95\% de los GT (Friedman, 2006). Esta situación ha llevado a establecer directrices que limitan su contenido en nuevas variedades, permitiéndose en la normatividad de algunos países niveles menores a 200 $\mathrm{mgkg}^{-1}$ papa fresca, valores superiores pueden llegar ser perjudiciales para la salud (Friedman, 2006; Milner et al., 2011; Tajner-Czopek et al. 2014; Cheng-Yu et al., 2018).

El efecto de los procesos tecnológicos sobre la a-solanina y a-chaconina ha sido muy debatido sin llegar a una conclusión determinante. Algunos autores han reportado una disminución durante las operaciones de pelado y cocción (Pęksa et al. 2006; Tajner-Czopek et al. 2008; Rytel, 2012), otros reportan una alta termorresistencia y estabilidad al calor por lo que no genera una disminución significativa en procesos de cocción, fritura y horneado (Griffiths y Dale, 2001), ni en proceso de cocción por microondas, debido a que los GT no son solubles en agua a pH neutro (Mulinacci et al. 2008). La fritura al vacío (FV) de alimentos es una tecnología que durante las últimas dos décadas ha venido ganando importancia, debido a las mejores propiedades en los productos freídos frente a los obtenidos en fritura convencional (fritura atmosférica) (Moreira, 2014). Sin embargo, la literatura no reporta investigaciones de la influencia del proceso de FV sobre el contenido de glicoalcaloides, se resaltan bondades de este proceso sobre los atributos de calidad y menor absorción de aceite (Crosa et al. 2014; Diamante et al., 2015; Belkova et al., 2018), mayor retención de la calidad nutricional (fitoquímicos) (Da Silva y Moreira, 2008; Fang, Z. et al., 2011) en alimentos como: zanahoria (Dueik et al., 2013), Banana (Sothornvit, 2011), papa (Yagua y Moreira, 2011) entre otros. El objetivo de la investigación fue evaluar la influencia de la FV sobre el contenido de glicoalcaloides $\alpha$-solanina y a-chaconina en chips de papa cv Botella Roja.

\section{METODOLOGÍA}

Se utilizaron papas variedad Botella Roja provenientes de la granja Experimental Botana de la Universidad de Nariño, Pasto, Colombia, altitud: 2820 m.s.n.m, temperatura: $\approx 12^{\circ} \mathrm{C}$, seccionadas y clasificadas de acuerdo la NTC 341, (2003). Para el proceso de FV se utilizaron rodajas con cáscara (espesor $2 \mathrm{~mm}$ ) y aceite $100 \%$ vegetal (Girasoli) proveniente de girasol, libre de grasas trans y con estabilidad al calor. La extracción de glicoalcaloides $\alpha$-solanina y $\alpha$-chaconina en papa se realizó de acuerdo a la metodología descrita por KarlErick, (1986) con modificaciones, donde inicialmente $3 \mathrm{~g}$ de muestra en estado fresco se trituró en un mortero y se le adicionó $60 \mathrm{~mL}$ de una mezcla cloroformo:metanol (2:1 V/V), luego se agitó a velocidad baja durante $10 \mathrm{~min}$, posteriormente se adicionó $60 \mathrm{~mL}$ de la mezcla cloroformo:metanol y se agitó a velocidad alta por una hora, se rotaevaporó a $60^{\circ} \mathrm{C}$ hasta concentrar a un volumen aproximado de $20 \mathrm{~mL}$. A este concentrado se le adicionó $9 \mathrm{~mL}$ de solución de $\mathrm{HCl}$ 0,2 N, agitándose por 20 min y filtrado posterior en papel Whatman No. 1. 
El extracto obtenido se adicionó $\mathrm{NH}_{4} \mathrm{OH} 25 \%$ hasta ajustar a un pH de 11, luego se calentó a $70^{\circ} \mathrm{C}$ por 20 min y se dejó en refrigeración $\left(4^{\circ} \mathrm{C}\right)$ por una hora, finalmente, se centrifugó a 7500 rpm por 20 min descartándose el sobrenadante.

El precipitado se disolvió en $5 \mathrm{~mL}$ de una solución de acetonitrilo:agua:buffer fosfato $\mathrm{pH}$ 7,6 (60:30:10 V/V/V), y la cuantificación $\alpha$-solanina y $\alpha$-chaconina se realizó por cromatografía líquida de alta resolución (HPLC) en fase reversa en un equipo Breeze 2 Waters, operado con el software Empower 2,0 (Waters, USA), bomba binaria 1525, detector PDA 2998, columna Thermo Scientific - Hypersil GOLD C18, $5 \mu \mathrm{m} \times 4,6 \mathrm{~mm} \times 250 \mathrm{~mm}$, flujo de 1,5 $\mathrm{mL}$ de fase móvil (acetonitrilo:agua:buffer fosfato $\mathrm{pH} 7,6)(60: 30: 10 \mathrm{~V} / \mathrm{V} / \mathrm{V})$, temperatura $40^{\circ} \mathrm{C}$, longitud de onda: $205 \mathrm{~nm}$ (AOAC 997.13, 2000). Se construyeron las curvas de calibración para ambos glicoalcaloides a concentraciones entre $0-50$ ppm con los estándares descritos en la tabla 1. Los contenidos de $\alpha$-solanina y $\alpha$-chaconina para papa fresca y snack se expresan en $\mathrm{mgkg}^{-1}$.

Tabla 1: Estándares de glicoalcaloides para la determinación del método

\begin{tabular}{|c|c|c|}
\hline Nombre & $\alpha$-solanina & $\alpha$-chaconina \\
\hline Número de producto & S3757 & $021553 \mathrm{~S}$ \\
\hline Lote & SLBS1906V & 16100715 \\
\hline Número CAS & $20562-01-1$ & $20562-03-2$ \\
\hline Pureza HPLC & $98 \%$ & $99,1 \%$ \\
\hline Fórmula & $\mathrm{C}_{45} \mathrm{H}_{73} \mathrm{NO}_{15}$ & $\mathrm{C}_{45} \mathrm{H}_{73} \mathrm{NO}_{14}$ \\
\hline Peso & $868,06 \mathrm{gmol}^{-1}$ & 852,08 gmol $^{-1}$ \\
\hline Marca & SIGMA-ALDRICH & INDOFINE Chemical Company, Inc. \\
\hline
\end{tabular}

La estandarización del método de cuantificación de los GT se realizó a través de la metodología descrita por Duffau et al. (2010): linealidad, precisión, sensibilidad, límite de detección y límite de cuantificación. La precisión se cuantificó como coeficiente de variación a una concentración de 0,5 ppm, la sensibilidad corresponde a la pendiente de la recta de calibración, el límite de detección (LD) se realizó a partir de seis mediciones de una concentración estimada $(0,5 \mathrm{ppm})$ cercana al blanco, según la ecuación $\mathrm{LD}=2^{*} \mathrm{P}^{*}$ So, donde: $P=$ probabilidad t Student ( 1 - $\alpha=$ significancia estadística; $v=$ grados de libertad) y So= desviación estándar. El límite de cuantificación (LC) se determinó bajo las condiciones de operación del método seleccionado y de acuerdo a la ecuación $\mathrm{LC}=10$ So.

La evaluación de la influencia de la FV sobre el contenido de glicoalcaloides ( $\alpha$-solanina y $\alpha$-chaconina) en los chips de papa, se realizó utilizando la metodología de superficie de respuesta con un diseño central compuesto cara centrada $(\alpha=1)$ con 15 experimentos, para un total de 75 muestras (5 muestras/experimento), considerándose las variables independientes: $\Delta T$ ( $T_{\text {aceite }}-T_{\text {ebullición agua })}\left(40-60{ }^{\circ} \mathrm{C}\right)$, presión de vacío (PV) (30 - $70 \mathrm{kPa}$ ) y tiempo (t) (3 - $7 \mathrm{~min}$ ). La optimización experimental del proceso con múltiples respuestas se realizó utilizando la función de deseabilidad bajo el criterio de minimizar los contenidos de las variables $\alpha$-solanina y a-chaconina; además, se compararon los valores experimentales a la condición óptima del proceso de FV frente a los valores predichos por el modelo y así, evaluar la efectividad de los modelos matemáticos obtenidos. Para el análisis de datos (ANOVA), los gráficos de superficie de respuesta y la optimización se utilizó el software STATGRAPHICS Centurión XVI Versión 16.1.03 (StatPoint Technologies, Inc. 1982-2010).

\section{RESULTADOS Y DISCUSIÓN}

Los resultados de esta investigación se presentan en tres apartados: estandarización del método, glicoalcaloides en papa fresca, efecto de la fritura al vacío sobre los glicoalcaloides y optimización del proceso de fritura al vacío

\section{Estandarización del método}

La figura 1 presenta las curvas de calibración para la cuantificación por HPLC de $\alpha$-solanina y a-chaconina, las cuales denotan un buen ajuste de los datos en el rango evaluado, siendo la linealidad de 0,9977 y 0,9974 respectivamente. Las precisiones del $\alpha$-solanina y $\alpha$-chaconina presentaron bajos coeficientes de variabilidad: $14 \%\left(0,11 \mathrm{mgkg}^{-1}\right)$ y $13 \%\left(0,32 \mathrm{mgkg}^{-1}\right)$ respectivamente, lo que confiere una buena exactitud para su cuantificación. Por otro lado, la sensibilidad de ambos componentes fue relativamente similar: $2657,8 \mathrm{Uv} / \mathrm{s}$ 
ppm ( $\alpha$-solanina) y $2725,5 \mathrm{Uv} / \mathrm{s}$ ppm ( $\alpha$-chaconina); mientras que los LD fueron 0,35 y 1,04 ppm y los LC 1,07 y 3,17 ppm respectivamente. En general los resultados obtenidos dan confianza a los resultados presentados.

\section{Glicoalcaloides en papa fresca}

La papa cv Botella Roja presentó un contenido de $\alpha$-solanina de 22,8 $\pm 2,4 \mathrm{mgkg}^{-1}$ papa y $\alpha$-chaconina $27,6 \pm$ $1,9 \mathrm{mgkg}^{-1}$ papa, con un GT de 50,43 $\mathrm{mgkg}^{-1}$ papa. La literatura reporta en diferentes variedades valores próximos a los encontrados: Tajner-Czopek et al. (2014) (GT =61,1 $\mathrm{mgkg}^{-1}$ papa var. Blue Congo y 40,5 $\mathrm{mgkg}^{-1}$ papa var. Vitelotte), Rytel, (2012) (GT = $32 \mathrm{mgkg}^{-1}$ papa var. Karlena). En general, la concentración de GT depende de diversos factores: variedad, condiciones de cultivo, fertilización nitrogenada, condiciones climáticas y almacenamiento de los tubérculos (Tajner-Czopek et al., 2008; Rytel, 2012; Amer et al., 2014). Varios autores afirman que la cantidad de $\alpha$-chaconina es mayor que la $\alpha$-solanina (Friedman, 2006; Knuthsen et al., 2009).
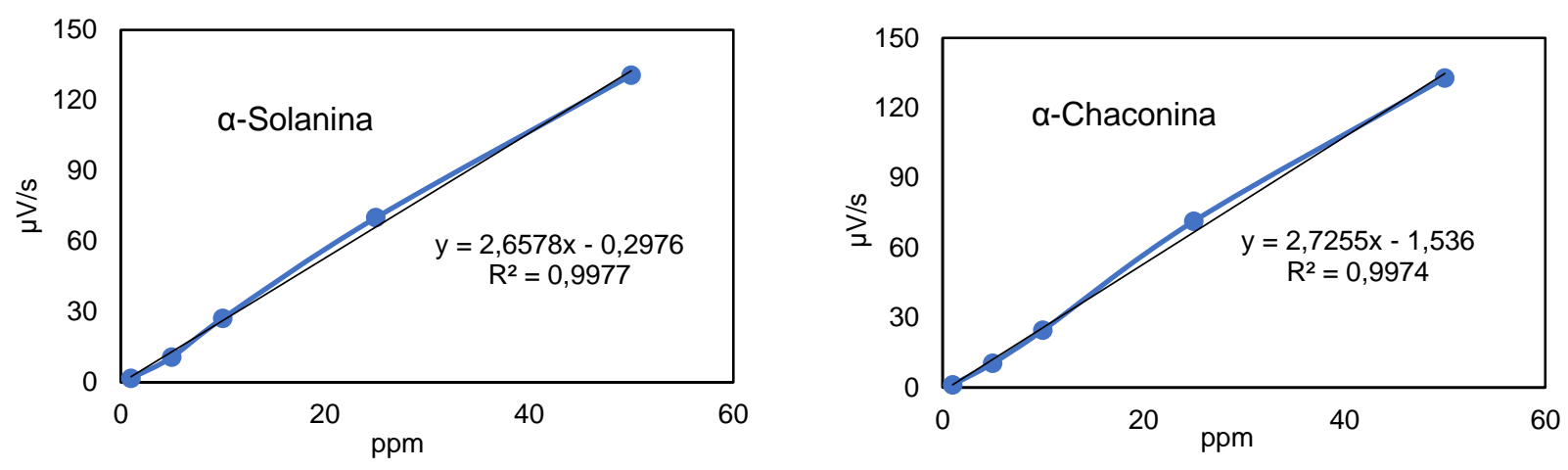

Fig. 1: Curva de calibración de $\alpha$-solanina y $\alpha$-chaconina (área en $\mu \mathrm{V} / \mathrm{s}$ en miles).

\section{Efecto de la fritura al vacío sobre los glicoalcaloides}

Las tablas 2 y 3 presentan los contenidos de $\alpha$-solanina y $\alpha$-chaconina en snack de papa cv Botella Roja y el ANOVA respectivamente. Por otro lado, la figura 2 presenta los gráficos de superficie de respuesta del $\alpha$ solanina y $\alpha$-chaconina en función de las variables independientes o factores. La $\alpha$-solanina en los snacks de papa presentó fluctuaciones de los valores medios entre 0 y $16,95 \mathrm{mgkg}^{-1}$ (disminución entre 0 y $100 \%$ con respecto a la papa fresca); mientras que, la $\alpha$-chaconina fluctuó entre 1,04 y 27,22 $\mathrm{mgkg}^{-1}$ (disminución entre 0 y $96 \%$ con respecto a la papa fresca). Esta disminución es atribuible a la descomposición de los GT muy probablemente a los productos de hidrólisis ( $\alpha$-chaconina $\rightarrow \beta 1$-chaconina $\rightarrow \beta_{2}$-chaconina $\rightarrow \gamma$-chaconina $\rightarrow$ solanidina y $\alpha$-solanina $\rightarrow \beta 1$ - solanina $\rightarrow \beta_{2}$ - solanina $\rightarrow \gamma$-solanina $\rightarrow$ solanidina), sustancias menos tóxicas que la forma $\alpha$, principalmente aquellos que se encuentran en la cáscara (Singh y Kaur, 2016). Esta situación confiere una influencia de las variables independientes evaluadas durante el proceso de FV; además de otros factores asociados a la producción primaria: variedad, suelo, fertilización, clima, agua, entre otros (Kozukue et al., 2008; Jensen et al., 2009).

El comportamiento del $\alpha$-solanina según los gráficos de superficie de respuesta, no muestra una tendencia bien definida de la influencia del $\Delta T$ ni del tiempo de fritura, si no que depende principalmente de las interacciones $\Delta T$ y tiempo y de la PV-tiempo, pero si se observa una disminución del a-solanina con el incremento de la presión en el sistema (PV altas). Se destaca que, los mínimos niveles o la no presencia de a-solanina en los snacks se alcanzan bajo combinaciones: $\Delta \mathrm{T}\left(46^{\circ} \mathrm{C}\right)$ y tiempo $(3 \mathrm{~min}), \mathrm{PV}(70 \mathrm{kPa})$ y tiempo (3-4 $\mathrm{min}) \circ \Delta \mathrm{T}\left(46^{\circ} \mathrm{C}\right)$ y $\mathrm{PV}(70 \mathrm{kPa})$. En cuanto a la $\alpha$-chaconina, los gráficos de superficie de respuesta muestran una disminución del contenido de a-chaconina con la disminución de la PV (mayor vacío en el sistema). La menor concentración de $\alpha$-chaconina en los ensayos experimentales fue de $1,05 \pm 1,57 \mathrm{mgkg}^{-1}$, obteniéndose a $\mathrm{PV}(50 \mathrm{kPa}), \Delta \mathrm{T}\left(50^{\circ} \mathrm{C}\right)$ y $\mathrm{t}(5 \mathrm{~min})$; sin embargo, el ANOVA mostró una interacción importante $\Delta T$-PV y $\Delta T$ - tiempo, donde los mínimos niveles o la no presencia de a-chaconina en los snacks se alcanzan bajo combinaciones: $\Delta \mathrm{T}(40-60 \stackrel{\circ}{\circ})$ - $\mathrm{PV}(40 \mathrm{kPa}), \Delta \mathrm{T}(40 \stackrel{\circ}{\circ} \mathrm{C})-\mathrm{t}(5 \mathrm{~min})$ y $\Delta \mathrm{T}\left(52{ }^{\circ} \mathrm{C}\right)-\mathrm{t}(3 \mathrm{~min})$. Para determinar si las medias de los contenidos de glicoalcaloides con respecto al tratamiento aplicado son significativamente diferentes se empleó el procedimiento de diferencia mínima significativa (LSD) de Fisher al $95 \%$, con este procedimiento para a-solanina y a-chaconina se identificaron 8 y 4 grupos homogéneos según la coincidencia de las letras (Tabla 2). No existen diferencias estadísticamente significativas entre aquellos experimentos que compartan una misma letra. 
La disminución de GT de productos procesados térmicamente con respecto a papas frescas, ha sido reportada por diversos autores: Tajner-Czopek et al. (2014) reportaron una disminución del $97 \%$ después de un proceso de fritura atmosférica de papa var. Highland Burgundy Red y Mäder et al. (2009) reportaron una disminución del $25 \%$ después de someter a vapor papas del cultivar Karlena. Otros autores reportaron una disminución del $15 \%$ con tratamiento en microondas; mientras que, en procesos de fritura atmosférica los GT variaron de acuerdo con la temperatura de fritura, no observándose afectación a $157^{\circ} \mathrm{C}$; mientras que, a 210 ${ }^{\circ} \mathrm{C}$ los alcaloides se descomponen parcialmente después de 10 min de calentamiento: $\alpha$-chaconina $(64,9 \%)$ y $\alpha$-solanina (59,7\%) (Takagi et al., 1990).

Tabla 2: Promedio, desviación estándar y prueba múltiple de rangos - LSD del contenido de GT en snack de papa cV Botella Roja. *Letras iguales corresponden al mismo grupo homogéneo

\begin{tabular}{|c|c|c|c|c|c|c|c|}
\hline Experimento & $\begin{array}{c}\text { Factor } A \\
\Delta T \\
\left.{ }^{\circ} \mathrm{C}\right)\end{array}$ & $\begin{array}{c}\text { Factor } B \\
P V \\
(\mathrm{kPa})\end{array}$ & $\begin{array}{c}\text { Factor C } \\
(\mathrm{min})\end{array}$ & $\begin{array}{c}\alpha \text {-solanina } \\
\left(\mathrm{mgkg}^{-1}\right)\end{array}$ & $\begin{array}{c}\text { Prueba Múltiple } \\
\text { de Rangos- } \\
\text { LSD para } \\
\alpha \text {-solanina* }^{*}\end{array}$ & $\begin{array}{c}\alpha \text {-chaconina } \\
\left(\mathrm{mgkg}^{-1}\right)\end{array}$ & $\begin{array}{c}\text { Prueba Múltiple } \\
\text { de Rangos - } \\
\text { LSD para } \\
\text {-chaconina* }^{*}\end{array}$ \\
\hline 1 & 50 & 50 & 5 & $3,38 \pm 1,17$ & $\mathrm{DE}$ & $4,34 \pm 1,11$ & $\mathrm{~A}$ \\
\hline 2 & 50 & 30 & 5 & $16,95 \pm 1,88$ & $\mathrm{H}$ & $12,74 \pm 2,57$ & $\mathrm{~B}$ \\
\hline 3 & 50 & 50 & 3 & $4,06 \pm 1,41$ & $\mathrm{E}$ & $4,34 \pm 2,73$ & $\mathrm{~A}$ \\
\hline 4 & 60 & 70 & 3 & $7,39 \pm 1,54$ & $\mathrm{~F}$ & $11,23 \pm 2,43$ & $\mathrm{~B}$ \\
\hline 5 & 50 & 50 & 5 & $0,55 \pm 0,81$ & $\mathrm{AB}$ & $1,04 \pm 1,56$ & $\mathrm{~A}$ \\
\hline 6 & 50 & 50 & 5 & $0,73 \pm 1,25$ & $\mathrm{AB}$ & $1,23 \pm 2,21$ & $\mathrm{~A}$ \\
\hline 7 & 60 & 50 & 5 & $8,85 \pm 1,30$ & $\mathrm{G}$ & $10,42 \pm 2,15$ & $\mathrm{~B}$ \\
\hline 8 & 40 & 50 & 5 & No detectable & $\mathrm{A}$ & $13,76 \pm 2,39$ & $\mathrm{BC}$ \\
\hline 9 & 40 & 30 & 3 & $3,68 \pm 0,43$ & $\mathrm{DE}$ & $16,29 \pm 2,24$ & $\mathrm{C}$ \\
\hline 10 & 60 & 30 & 7 & $2,61 \pm 0,67$ & $\mathrm{CD}$ & $25,70 \pm 5,79$ & $\mathrm{D}$ \\
\hline 11 & 50 & 70 & 5 & $3,00 \pm 0,79$ & $\mathrm{CDE}$ & $26,20 \pm 3,26$ & $\mathrm{D}$ \\
\hline 12 & 40 & 70 & 7 & $3,26 \pm 0,42$ & $\mathrm{DE}$ & $27,22 \pm 4,50$ & $\mathrm{D}$ \\
\hline 13 & 50 & 50 & 5 & $1,86 \pm 0,24$ & $\mathrm{BC}$ & $3,29 \pm 0,65$ & $\mathrm{~A}$ \\
\hline 14 & 50 & 50 & 7 & $1,16 \pm 1,19$ & $\mathrm{AB}$ & $1,90 \pm 1,78$ & $\mathrm{~A}$ \\
\hline 15 & 50 & 50 & 5 & $3,96 \pm 0,87$ & $\mathrm{E}$ & $3,418 \pm 0,59$ & $\mathrm{~A}$ \\
\hline
\end{tabular}

Tabla 3: ANOVA para contenido de GT en snack de papa. * Significativo $(p<0,05)$

\begin{tabular}{|c|c|c|c|}
\hline \multicolumn{2}{|c|}{ Variables } & $\alpha$-solanina & $\alpha$-chaconina \\
\hline \multirow{4}{*}{$\begin{array}{c}\text { Efectos } \\
\text { Factores } \\
\text { principales }\end{array}$} & Factor A: $\Delta \mathrm{T}$ & $0,00^{*}$ & 0,10 \\
\cline { 2 - 4 } & Factor B: PV & $0,00^{*}$ & $0,00^{*}$ \\
\cline { 2 - 4 } & Factor C: tiempo & $0,03^{*}$ & 0,23 \\
\hline \multirow{4}{*}{$\begin{array}{c}\text { Interacciones } \\
\text { cuadráticas }\end{array}$} & $\mathrm{AA}$ & 0,21 & $0,00^{*}$ \\
\cline { 2 - 4 } & $\mathrm{BB}$ & $0,00^{*}$ & $0,00^{*}$ \\
\cline { 2 - 4 } & $\mathrm{CC}$ & $0,00^{*}$ & $0,00^{*}$ \\
\hline \multirow{3}{*}{$\begin{array}{c}\text { Interacciones } \\
\text { lineales }\end{array}$} & $\mathrm{AB}$ & 0,85 & $0,00^{*}$ \\
\cline { 2 - 4 } & $\mathrm{AC}$ & $0,00^{*}$ & $0,00^{*}$ \\
\cline { 2 - 4 } & $\mathrm{BC}$ & $0,00^{*}$ & 0,98 \\
\hline
\end{tabular}

Existen algunas consideraciones que atribuyen la disminución de los glicoalcaloides: Haase (2008) y Rytel (2012) lo asocian principalmente al tipo de proceso térmico aplicado sobre la matriz alimentaria; Mäder et al. (2009) reportaron que el pelado y escaldado son los principales causantes de las pérdidas de glicoalcaloides, 
mientras que la cocción, el macerado y el secado no tienen un efecto significativo en la disminución; Haddadin et al. (2001) mencionan que los glicoalcaloides no se destruyen durante los procesos de cocción, horneado y freído; Pęksa et al. (2006) reportaron que la mayor disminución ocurre en los procesos de pelado, corte, lavado y fritura, donde los chips obtenidos de la var. Karlena y Saturna contenían el $14 \%$ y $18 \%$ del estado en fresco respectivamente; finalmente, Haase (2008) reporta una temperatura crítica de $170{ }^{\circ} \mathrm{C}$ donde ocurre la descomposición de los dos principales alcaloides.

Otras investigaciones han reportado otras consideraciones importantes: Haddadin et al. (2001) reportaron que la cáscara de las papas es una fuente importante de $\alpha$-chaconina y $\alpha$-solanina; sin embargo, en los procesos de fritura, generalmente se realiza un pelado previo, disminuyendo la presencia de GT, los cuales son metabolitos esteroidales secundarios tóxicos, y a altas concentraciones tiene un efecto de la actividad anticolinesterasa y disrupción de la membrana celular (Nema et al., 2008). Por otro lado, Rytel (2012) encontró en procesos de deshidratación, que etapas previas de pelado y escaldado reducen los GT en un 50\%; mientras que, el producto deshidratado contiene un $14 \%$ de los GT iniciales.

\section{Optimización del proceso de fritura al vacío.}

La optimización experimental del proceso de FV se realizó bajo los criterios de minimizar los contenidos de $\alpha$ solanina y $\alpha$-chaconina y considerando iguales valores de impacto y peso para cada una de estas variables. La optimización estadística múltiple fijó las siguientes condiciones de proceso: $\Delta \mathrm{T}: 48,2^{\circ} \mathrm{C}\left(\mathrm{T}_{\text {aceite }}=131,4 \stackrel{\circ}{ } \mathrm{C}\right)$, PV: $49,0 \mathrm{kPa}$ y t: $7 \mathrm{~min}$, con una deseabilidad del 98,9\% y valores teóricos determinados por los modelos matemáticos de $-0,74 \mathrm{mgkg}^{-1}$ para $\alpha$-solanina y $-2,90 \mathrm{mgkg}^{-1}$ para $\alpha$-chaconina y los valores experimentales obtenidos a partir de 5 réplicas realizadas a las condiciones de proceso óptimas, presentaron valores de $1,4 \pm 1,3 \mathrm{mgkg}^{-1}$ y no detectable por el instrumento respectivamente, existiendo una buena aproximación entre lo teórico y experimental o un error residual bajo.
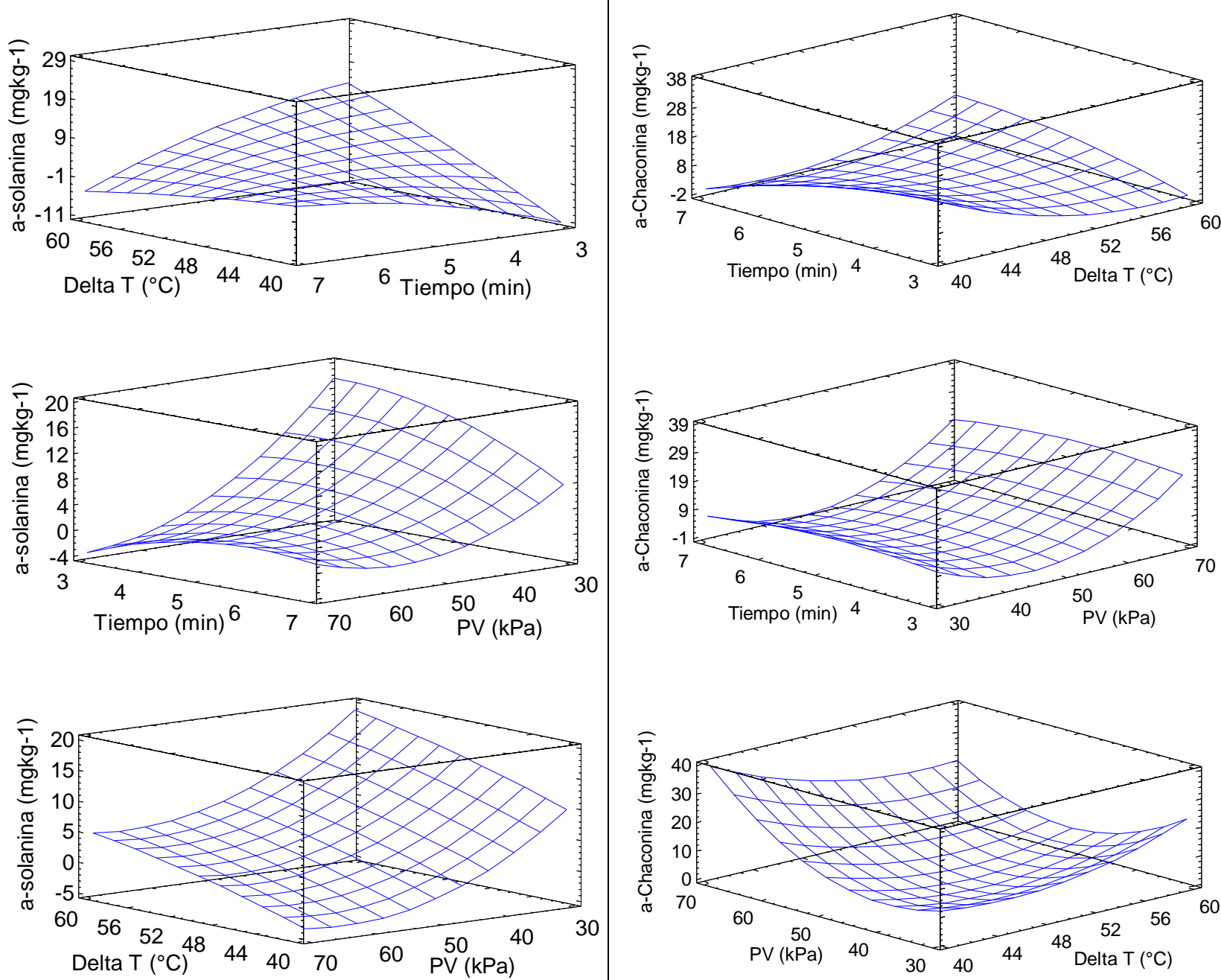

Fig. 2: Superficie de respuesta para $\alpha$-solanina y $\alpha$-chaconina 


\section{CONCLUSIONES}

A través del presente trabajo, se pueden extraer las siguientes conclusiones principales:

El proceso de FV representa una tecnología efectiva para la disminución del contenido de GT ( $\alpha$-solanina y a-chaconina) presentes en los snacks de papa cv Botella Roja con respecto al tubérculo fresco.

El contenido de GT está afectado por las variables independientes: $\Delta T$ ( $T$ aceite - Tevaporación agua), PV y tiempo de fritura; así como, las interacciones cuadráticas y sus interacciones lineales.

La etapa de optimización experimental permitió obtener la mayor disminución del contenido de $\alpha$-solanina $(96,2 \%)$ y $\alpha$-chaconina $(100 \%)$ a las condiciones de operación: $\Delta \mathrm{T}: 48,2{ }^{\circ} \mathrm{C}\left(\mathrm{T}_{\text {aceite }}=131,4{ }^{\circ} \mathrm{C}\right), \mathrm{PV}: 49,0 \mathrm{kPa}$ y t: 7 min, con una deseabilidad del $98,9 \%$.

La papa fresca no necesita una etapa de pelado previo cuando se desee procesar por FV. Los resultados obtenidos en la investigación presentaron una disminución similar a los reportados por otros estudios en diferentes variedades de papa y en otros procesos de cocción.

\section{AGRADECIMIENTOS}

La investigación se realizó en el marco del proyecto "Mejoramiento Tecnológico y Productivo del Sistema Papa en el Departamento de Nariño", financiado por el Sistema General de Regalías FCTel - Gobernación de Nariño, con el apoyo del Grupo de Investigación de Alimentos Funcionales (GAF) de La Universidad Nacional de Colombia y el Grupo de Investigación Tecnologías Emergentes en Agroindustria de La Universidad de Nariño.

\section{REFERENCIAS}

Amer, F.S., L. Reddivari y otros cuatro autores, Effect of Genotype and Storage on Glycoalkaloid and Acrylamide Content and Sensory Attributes of Potato Chips, doi: 10.1007/s12230-014-9393-9, Am. J. Potato Res., 91(6), 632-641 (2014)

AOAC, Official Methods of Analysis, 997.13. Glycoalkaloids (a-Solanine and a-Chaconine) in Potato Tubers. AOAC INTERNATIONAL (2000)

Belkova, B., J. Hradecky y otros cuatro autores, Impact of Vacuum Frying on Quality of Potato Crisps and Frying Oil, doi: 10.1016/j.foodchem.2017.08.062, Food Chemistry, Elsevier, 241, 51-59 (2018).

Cheng-Yu, J., L. Hong y otros cinco autores, Glycoalkaloids and Phenolic Compounds in Three Commercial Potato Cultivars Grown in Hebei, China, doi: 10.1016/j.fshw.2018.02.00, Food Science and Human Wellness, 7, 156-162 (2018)

Crosa, M.J., V. Skerl y otros cinco autores, Changes Produced in Oils During Vacuum and Traditional Frying of Potato Chips, doi: 10.1016/j.foodchem.2013.08.13, Food Chemistry, Elsevier Ltd, 146(2), 603-607 (2014)

Da Silva, P.F. y R.G. Moreira, Vacuum Frying of High-quality Fruit and Vegetable-Based Snacks, doi: 10.1016/j.Iwt.2008.01.016, LWT - Food Science and Technology, 41(10), 1758-1767 (2008)

Diamante, L.M., S. Shi, A. Hellmann y J. Busch, Vacuum Frying Foods: Products, Process and Optimization, International Food Research Journal, 22(1), 15-22 (2015)

Dueik, V., C. Marzullo y P. Bouchon, Effect of Vacuum Inclusion on the Quality and the Sensory Attributes of Carrot Snacks, doi: 10.1016/j.Iwt.2012.05.011, LWT - Food Science and Technology, Elsevier Ltd, 50(1), 361-365 (2013)

Duffau, B. y F, Rojas y otros seis autores, Validación de Métodos y Determinación de la Incertidumbre de la Medición: "Aspectos Generales Sobre la Validación de Métodos", Instituto de Salud Pública de Chile, 70 p., Chile (2010)

Fang, Z., D. Wu y otros cuatro autores, Phenolic Compounds in Chinese Purple Yam and Changes During Vacuum Frying, doi: 10.1016/j.foodchem.2011.03.123, Food Chemistry, Elsevier Ltd, 128(4), 943-948 (2011)

FAO, Organización de las Naciones Unidas para la Agricultura y la Alimentación, FAOSTAT. Disponble en: http://www.fao.org/faostat/es/\#data (2017)

FEDEPAPA, Federación Colombiana de Productores de Papa, Disponible en: http://fedepapa.com/wpcontent/uploads/2016/01, (2016)

Friedman, M., Potato Glycoalkaloids and Metabolites: Roles in the Plant and in the Diet, doi: 10.1021/jf061471t, Journal of Agricultural and Food Chemistry, 54(23), 8655-8681 (2006)

Griffiths, D.W. y M.F. Dale, Effect of Light Exposure on the Glycoalkaloid Content of Solanum Phureja Tubers, doi: 10.1021/jf010656r, Journal of Agricultural and Food Chemistry, 49(11), 5223-5227 (2001)

Haase, N., Healthy Aspects of Potatoes as Part of the Human Diet, doi: 10.1007/s11540-008-9111-4, Potato Research, $51(3), 239-258$ (2008) 
Haddadin, M., M. Humeid, F. Qaroot y R. Robinson, Effect of Exposure to Light on the Solanine Content of Two Varieties of Potato (Solanum tuberosum) Popular in Jordan, doi: 10.1016/S0308-8146(00)00279-X, Food Chemistry, 73(2), 205208 (2001)

Jensen, P.H., O.S. Jacobsen y otros tres autores, Degradation of the Potato Glycoalkaloids a-Solanine and $\alpha$-Chaconine in Groundwater, doi: 10.1007/s00128-009-9698-4, Bull Environ Contam Toxicol, 82, 668-672 (2009)

Karl-Erik, H., A Simplified Procedure for Quantification of Potato Glycoalkaloids in Tuber Extracts by H.P.L.C. Comparison with ELISA and a Colorimetric Method, doi: 10.1002/jsfa.2740370810J, Journal of the Science of Food and Agriculture, 37(8), 776-782 (1986)

Knuthsen, P., U. Jensen, B. Schmidt e I. Krog, Glycoalkaloids in potatoes: Content of Glycoalkaloids in Potatoes for Consumption, doi: 10.1016/j.jfca.2008.10.003, Journal of Food Composition and Analysis, 22(6), 577-581 (2009)

Kozukue, N., K.S. Yoon y otros cuatro autores, Distribution of Glycoalkaloids in Potato Tubers of 59 Accessions of Two Wild and Five Cultivated Solanum Species, doi: 10.1021/jf802631t, Journal of Agricultural and Food Chemistry, 56(24), 11920-11928 (2008)

Latorre-Vásquez, L., L. Córdoba-Solarte y otros cuatro autores, Elaboración de una Escala para Determinar Forma en Tubérculos de Papa Nativa, doi: 9789942287953 . VII Congreso Ecuatoriano de La Papa - Adaptación Al Cambio Climatico, 171-172, Tulcan, Ecuador 29 de Junio - 1 de julio (2017)

Mäder, J., H. Rawel y L.W. Kroh, Composition of Phenolic Compounds and Glycoalkaloids Alpha-Solanine and AlphaChaconine During Commercial Potato Processing, doi: 10.1021/jf901066k, Journal of Agricultural and Food Chemistry, 57(14), 6292-6297 (2009)

Milner, S.E., N.P. Brunton y otros cuatro autores, Bioactivities of Glycoalkaloids and Their Aglycones from Solanum Species, doi: 10.1021/jf200439q, Journal of Agricultural and Food Chemistry, 59(8), 3454-3484 (2011)

Moreira, R. G., Vacuum Frying Versus Conventional Frying - An overview, doi: 10.1002/ejlt.201300272, European Journal of Lipid Science and Technology, 116, 723-734 (2014)

Mulinacci, N., F. lere y otros seis autores, Effect of Cooking on the Anthocyanins, Phenolic Acids, Glycoalkaloids, and Resistant Starch Content in Two Pigmented Cultivars of Solanum tuberosum L., doi: 10.1021/jf801521e, Journal of Agricultural and Food Chemistry, 56(24), 11830-11837 (2008)

Nema, P.K., N. Ramayya, E. Duncan y K. Niranjan, Potato Glycoalkaloids: Formation and Strategies for Mitigation, doi: 10.1002/jsfa.3302, Journal of the Science of Food and Agriculture, 88(11), 1869-1881 (2008)

Pęksa, A., G. Gołubowska y otros tres autores, Changes of Glycoalkaloids and Nitrate Contents in Potatoes During Chip Processing, doi: 10.1016/j.foodchem.2005.03.035, Food Chemistry, 97(1), 151-156 (2006)

Rytel, E., Changes in the Levels of Glycoalkaloids and Nitrates After the Dehydration of Cooked Potatoes, doi: 10.1007/s12230-012-9273-0, American Journal of Potato Research, 89(6), 501-507 (2012)

Rytel, E., The Effect of Industrial Potato Processing on the Concentrations of Glycoalkaloids and Nitrates in Potato Granules, doi: 10.1016/j.foodcont.2012.04.049, Food Control, 28(2), 380-384 (2012)

Singh, J. y L. Kaur, Advances in Potato Chemistry and Technology, ISBN: 978-0-12-800002-1 Copyright @ Elsevier Inc. (2016)

Sothornvit, R., Edible Coating and Post-frying Centrifuge Step Effect on Quality of Vacuum-fried Banana Chips, doi: 10.1016/j.jfoodeng.2011.07.010, Journal of Food Engineering Elsevier Ltd, 107(3-4), 319-325 (2011)

Tajner-Czopek, A., M. Jarych-Szyszka y G. Lisińska, Changes in glycoalkaloids content of potatoes destined for consumption, doi: 10.1016/j.foodchem.2007.06.034, Food Chemistry, 106(2), 706-711 (2008)

Tajner-Czopek, A., E. Rytel, M. Aniołowska y K. Hamouz, The Influence of French Fries Processing on the Glycoalkaloid Content in Coloured-Fleshed Potatoes, doi: 10.1007/s00217-014-2163-6, European Food Research and Technology, 238(6), 895-904 (2014)

Takagi, K., M. Toyoda, Y. Fujiyama y Y. Saito, Effect of Cooking on Contents of $\alpha$-Chaconine and a-Solanine in Potatoes, Journal of the Food Hygienic Society of Japan, Shokuhin Eiseigaku Zasshi, 31(1), 67-73 (1990)

Tömösközi-Farkas, R., H. Daood, Z. Polgár y G. Hajós, Determination of Glycoalcaloids in Hungarian Potatoes by HPLC, doi: 10.1365/s10337-006-0763-4, Chromatographia, 63, 115-118 (2006)

Yagua, C.V. y R.G. Moreira, Physical and Thermal Properties of Potato Chips During Vacuum Frying, doi: 10.1016/j.jfoodeng.2010.12.018, Journal of Food Engineering, Elsevier Ltd, 104(2), 272-283 (2011) 